\title{
L'invention de la sphère domestique au sortir de la révolution
}

Jennifer Heuer et Anne Verjus

\section{(2) OpenEdition \\ 1 Journals}

\section{Édition électronique}

URL : https://journals.openedition.org/ahrf/543

DOI : 10.4000/ahrf.543

ISSN : 1952-403X

Éditeur :

Armand Colin, Société des études robespierristes

Édition imprimée

Date de publication : 1 mars 2002

Pagination : 1-28

ISSN : 0003-4436

\section{Référence électronique}

Jennifer Heuer et Anne Verjus, "L'invention de la sphère domestique au sortir de la révolution »,

Annales historiques de la Révolution française [En ligne], 327 | janvier-mars 2002, mis en ligne le 19 mars 2008, consulté le 23 avril 2022. URL : http://journals.openedition.org/ahrf/543 ; DOI : https://doi.org/ 10.4000/ahrf.543 


\title{
L'INVENTION DE LA SPHÈRE DOMESTIQUE AU SORTIR DE LA RÉVOLUTION
}

\author{
JENNIFER HEUER \& ANNE VERJUS
}

\begin{abstract}
Les catégories juridiques et politiques de la Révolution française.font abstraction de la famille comme corps intermédiaire entre l'individu et l'État. Cette absence, d'abord non vue et non voùlue, a laissé la voie ouverte à des interprétations et des revendications qui, par les contradictions qu'elles ont fait émerger entre les membres de la famille, les membres de la nation et les membres du Souverain, ont obligé les législateurs à préciser un certain nombre de notions et à mettre en place une séparation stricte entre la société des citoyens et la famille. C'est ainsi qu'au sortir de la société d'Ancien Régime pensée comme une famille, sur la voie de laquelle les députés de l'Assemblée nationale s'étaient d'abord engagés, les problèmes juridiques nés de la contradiction entre les membres de la société et ceux de la famille ont contraint les révolutionnaires à mieux définir la nation, en l'abordant comme un agglomérat de familles.
\end{abstract}

Mots clés: droits des femmes; famille nationale; épouses d'émigrés; devoirs patriotiques; cité politique.

Vers la fin de l'année 1794 (1), alors que la politique de la Terreur n'en finit plus d'organiser sa propre fin, une mère supplie le gouvernement révolutionnaire de bien vouloir l'acquitter de sa fuite hors de France. Consciente des risques mortels que lui font encourir cette émigration dont elle récuse la responsabilité, la jeune femme tente d'amoindrir sa faute en l'imputant à son père et à son époux sous l'autorité desquels l'ancienne loi la plaçait et auxquels elle devait obéissance; par ailleurs, elle présente son retour vers son pays natal, et donc sa désobéissance filiale et conjugale, comme un acte de patriotisme :

«jai voyagée avec une permission de conseil executif, forcée, enchainée par la volontée dun pere dun epoux, trois fois jai resistée, jai refusée ce

(1) La pétition dont il est question n'est pas datée; mais les références que fait la jeune femme aux lois sur les émigrés font penser qu'elle l'a écrite aux alentours du mois de novembre 1794.

Annales historiques de la Révolution française $-2002-N^{\circ} 1$ [1 à 28] 
voyage, trois fois jen ai presse le retour, en fin j'ai desobie a la puissance paternelle et maritalle, je les a abandonnées pour obéir aux lois de mon pays. [...] "Je me suis dit, les prejuges, legoïsme, les loix abusives de notre ancien gouvernement sont changés. On ne devoit rien à sa patrie, maintenant comme a rome, on lui sacrifie son père son époux, et tous les etres qui ne doivent nous etres chers qu'apres elle." [...]” (2)

Nous ne savons pas ce qu'il advint de cette femme. Néanmoins, son cas entre dans le cadre de la loi qui prévoyait que la subordination familiale n'était en aucune façon une excuse recevable pour les émigrés; le devoir patriotique emportant tous les autres, ils n'étaient pas supposés invoquer leurs devoirs conjugaux, non plus que les enfants prétexter de leur piété filiale, pour justifier de leur fuite. Le gouvernement de la Terreur aura fait guillotiner bien des épouses, bien des fils et filles accusés de n'avoir pas su choisir à temps entre les devoirs contradictoires d'un lien familial qui, pensaient-ils, les protégerait peut-être, et d'un lien national dont ils ne s'étaient probablement pas pensés redevables, du moins jusqu'à leur retour sur le sol français. Reste que cette politique n'est pas uniquement imputable au gouvernement de la Terreur : en matière de fidélité patriotique, les lois ne changent guère entre le début et le mitan de la Révolution : ainsi, l'obligation de faire prévaloir l'appartenance nationale sur le lien familial prévaut-elle au moins pendant toute la première période (1789-1794) et concerne autant le citoyen que la citoyenne et leurs enfants, c'est-à-dire tous les membres de la famille. Une seule pensée semble guider le législateur pour juger alors des devoirs des personnes : la nation tout entière est une famille politique dans laquelle aucune préférence, aucune particularité statutaire, n'est censée prévaloir sur le dévouement de chacun à la chose publique.

Bien différente, la décennie qui succède à cette apogée de la «famille nationale » voit au contraire poindre les premiers éléments d'une distinction nette entre ce qui va devenir la «sphère domestique» et le reste de la société civile et politique, distinction qui trouve sa réalisation complète dans le Code civil et clôt pour longtemps l'époque des transformations en la matière. C'est encore une jeune femme qui, parmi bien d'autres, nous permet de prendre la mesure de ce renversement; son cas est l'illustration qui terminera ce préambule. Suzanne Lepeletier est tout le contraire de cette jeune émigrée accusée d'avoir fui son pays (3). Fille d'un conventionnel assassiné, orpheline de mère, elle a été adoptée par l'Assemblée comme fille de la nation, puis élevée par son oncle. Lorsque ce dernier,

(2) A.N., D III 238. Nous avons conservé l'orthographe d'origine.

(3) Voir l'analyse détaillée de ce procès célèbre dans Jennifer HEUER, "Adopted Daughter of the French People : Suzanne Lepeletier and her Father, the National Assembly ", dans French Politics, Culture, and Society, 17, $n^{\circ} 3$ et $n^{\circ} 4,1999$, pp. 31-51; ainsi que dans Jennifer HEUER, Foreigners, families and citizens : contradictions of national citizenship in France, 1789-1830, ph. D. discuss.,University of Chicago, Chicago, 1998, chapitre 4. 
accusé d'avoir pris part à la Conspiration de Babeuf, perd ses droits civils (donc ceux qu'il avait, en tant que tuteur, sur sa nièce), Suzanne, alors âgée de 15 ans, obtient son émancipation; peu de temps après, se voyant opposer à son projet de mariage un refus catégorique de son grand-père et de ses oncles, elle porte l'affaire devant un conseil de famille; celui-ci argue que l'Assemblée nationale, en tant que "père adoptif » de Suzanne, doit intervenir pour empêcher le mariage, et envoie à cet effet une pétition aux directeurs. Une commission est mise en place; un large débat s'ouvre de décembre 1797 à février 1798 au cours duquel est discutée, au-delà du cas particulier de la jeune fille, la question de l'adoption nationale, et au terme duquel Jean-Pierre Chazal, rapporteur de la Commission, dénie à la Nation la possibilité d'être autre chose qu'un "père honorifique»: ne pouvant ni décéder, ni léguer ses biens à ses enfants, n'ayant par conséquent pas le devoir de protéger son patrimoine (justification de la puissance paternelle), il/elle n'a aucune possibilité de s'opposer au mariage de l'un de ses « enfants ». Par conséquent, Suzanne se voit reconnaître la pleine capacité de décider seule de son mariage; autrement dit, et bien que fille de la patrie, elle a désormais un droit, indépendant de son appartenance nationale : celui d'être seule juge de son sort matrimonial. Suzanne bénéficie de ne plus être la fille de personne, membre d'aucune "famille ", donc libre et indépendante, car entre les pères et la patrie, c'est désormais une nette séparation des compétences qui prévaut.

C'est sur cette transformation du traitement politique des personnes (4) que nous voudrions mettre l'accent dans le cadre de cet article. Transformation lente, opérée sous la pression douce des contradictions nées d'un modèle monarchique de l'appartenance nationale / familiale appliqué à une société politique de citoyens égaux en droits. Ces contradictions, d'abord inaperçues des contemporains, les ont peu à peu amenés à réaliser que l'ensemble des personnes n'étaient pas placées de manière indifférenciée sous l'autorité et la protection de la nation souveraine, et à imaginer une configuration radicalement nouvelle: une sphère domestique juridiquement et politiquement distincte de la patrie, une communauté familiale séparée de la société civile.

Le fait que cette séparation soit une conséquence du processus révolutionnaire ne va pas de soi. Bien que reconnue comme un élément fondamental, essentiel, de la société démocratique, elle est en effet généralement située à l'origine de la Révolution - dans les deux sens, temporel et philosophique, du terme (5). L'histoire des femmes est tout particulièrement traver-

(4) Traitement politique, au sens où nous ne nous en tiendrons pas au traitement judiciaire dont ces deux cas sont représentatifs. Nous étendrons le champ d'investigation à l'ensemble de la législation sur l'état familial des personnes en tant que question politique.

(5) Cf. Jürgen HABERMas, L'espace public. Anchéologie de la publicité comme dimension constitutive de la société bourgeoise, Paris, Payot, 1978; Reinhardt KOSELLECK, Le règne de la critique, Paris, Éd. de Minuit, coll. arguments, 1979. 
sée de ce postulat qui permet d'expliquer la situation exceptionnelle qui leur est réservée : Rousseau n'avait-il pas déjà opéré cette rupture en opposant la sphère domestique et la sphère politique (6) ? Pourtant, c'est un postulat qui résiste mal à l'examen de la législation des premières années de la Révolution; il faut croire que les hommes de cette période euphorique ont fait fi du précepte de Rousseau puisqu'ils se sont empressés d'étendre l'égalité démocratique non seulement aux liens fraternels et filiaux, mais aussi conjugaux, c'est-à-dire à l'ensemble des liens familiaux, continuant ainsi de confondre, sur la lancée des pratiques d'Ancien Régime, la société civile et la société familiale. Sans même parler des comportements individuels de certaines citoyennes (qui, en se réclamant ostensiblement du Souverain et d'une participation politique effective, montrent que l'idée de leur nonappartenance à la nation est loin d'aller de soi), les législateurs n'ont-ils pas tenu, eux-mêmes, à ce que les membres de la famille fassent prévaloir leur fidélité patriotique sur toutes autres, au premier rang desquelles celle due à leurs parents, leur fratrie et leur conjoint? En matière d'appartenances réciproques des uns et des autres aux différentes sphères qui émergent des premières années de la Révolution, une certaine confusion règne qui mérite qu'on s'y attarde. C'est cette persistance de l'identité entre liens de famille et liens politiques que nous traiterons en premier lieu.

\section{La « famille nationale » : persistance d'une notion}

En tentant de modifier le contenu des droits dans la famille pour les conformer aux principes de la société civile et politique des individus, les révolutionnaires continuent, dans un premier temps, et comme sous l'Ancien Régime, de considérer celle-ci comme une société politique (7). La différence majeure c'est que, liant entre elles des personnes libres et égales, la famille est désormais pensée et construite comme une société contractuelle, à l'image de la "grande " société; à ce titre, elle n'est pas une société spécifique : au contraire, elle disparaît dans la législation "intermédiaire» (8) au profit de l'universelle catégorie de l'individu. Absente du

(6) Cf. Carole Pateman, The Sexual Contract, Stanford, Stanford University Press, 1988; Joan B. LANDES, Women and the Public Sphere in the Age of the French Revolution, Cornell University Press, Ithaca and London, 1988; ainsi que Geneviève FRAISSE, Muse de la raison. La démocratie exclusive et la différence des sexes, Aix-en-Provence, Alinéa, 1989. C'est aussi l'analyse de Pierre Rosanvallon, Le sacre du citoyen. Histoire du suffrage universel en France, Paris, Gallimard, 1992.

(7) Les théories de la famille comme origine de la société politique sont assez bien connues pour ne pas nécessiter un retour sur cet aspect de la pensée d'Ancien Régime. Voir notamment Michel BorGETTO, "Métaphore de la famille et idéologies", Le droit non civil de la famille, PUF, 1983; ainsi que Christian BRUSCHI, «Essai sur un jeu de miroir : Famille / État dans l'histoire des idées politiques », dans L'État, la Révolution française et l'Italie, Presses universitaires d'Aix-Marseille, 1990.

(8) Qualificatif donné à la législation révolutionnaire pour mieux souligner son caractère de transition entre celle de l'Ancien Régime et celle du Code civil de 1804. 
vocabulaire des légistes révolutionnaires qui ne la définissent jamais, elle semble bien n'être plus considérée que comme une simple association, ou pour mieux dire, une "petite République ». Conjointement, la nation reste, toujours dans la lignée des pratiques et catégories juridiques d'Ancien Régime, une grande famille dont les membres peuvent être protégés, mais dont ils sont aussi redevables.

\subsection{La famille est une petite République...}

Une des premières lois de la Rẹvolution touchant à la famille concerne ainsi la protection de la liberté des enfants majeurs; c'est la loi des 16-24 août 1790 qui abolit les lettres de cachet (9); leur succèdent des « tribunaux de famille » qui imposent une décision collective de la famille sur les sujets de plainte et qui protègent les individus de l'arbitraire familial « en remettant entre les mains de la justice étatique l'exécution de la peine et la vérification de ses motifs» (10). C'est pour «faire de la famille une association régie comme le corps politique par la liberté et l'égalité, indépendante, d'ailleurs, de l'État, et en cela une association unique et privilégiée parce qu'elle est la seule naturelle " (11) que sont créés ces tribunaux de famille. D'une manière générale, la législation révolutionnaire va répondre à cet impératif politique de répandre la liberté et l'égalité jusque dans la société familiale des individus.

\section{Le mariage comme simple contrat}

Dans la Constitution de 1791 est indiqué, à l'article 7, que « la loi ne considère le mariage que comme un contrat civil». À l'instar de tous «les contrats de société parmi les hommes », le mariage doit être envisagé désormais comme une convention entre deux personnes, dont le consentement devrait seul suffire à produire les effets. Le mariage est à tel point conformé aux principes qui régissent les contrats que sa forme solennelle peut ne pas être respectée et le contrat demeurer implicite, du moment que se conjuguent les libres volontés des contractants (12).

(9) Cf. Arlette FARGE et Michel FouCAult, Le désordre des familles. Lettres de cachet des Archives de la Bastille, Paris, Gallimard / Julliard, 1982.

(10) Pierte MuRat, « La puissance paternelle et la Révolution française : essaj de régénération de l'autorité des pères ", dans La famille, la loi, l'État, de la Révolution au Code civil, Textes réunis et présentés par Irène Théry et Christian Biet, Paris, Imprimerie nationale, Centre Georges Pompidou, 1989, p. 400.

(11) Philippe SAGNAC, La législation civile de la Révolution française (1789-1804), essai d'histoire sociale, Paris, 1898 , p. 305.

(12) Voir les lumineuses remarques de Jacques Mulliez sur cet aspect peu connu du droit intermédiaire, dans "Pater is est...". La source juridique de la puissance paternelle du droit révolutionnaire au Code civil ", dans La famille, la loi, l'État..., op. cit., pp. 417-419. 
C'est au nom de cette liberté que les révolutionnaires, toujours à la veille de la proclamation de la République - le 20 septembre 1792 - votent la loi sur le divorce (13).

Enfin, ultime conséquence de cette volonté d'étendre la liberté dans tous les rapports de société, on remarquera l'absence, dans les trois projets de Code civil de Cambacérès, de toute obligation de fidélité, d'assistance et même de communauté de domicile, entre les époux. L'adultère n'est pas réprimé par le Code pénal de 1791, et n'est pas mentionné non plus dans la loi du 20 septembre 1792 parmi les causes de divorce; pour la première fois depuis le XIIe siècle, les relations personnelles entre époux sont laissées sous leur responsabilité (14). Le mariage est donc un contrat "comme les autres ", laïc et révocable.

\section{La famille, une association d'individus libres et égaux}

Légalité qui règne dans la société politique s'étend, comme la liberté, à la société familiale. En réalité, c'est moins la famille qui est visée par les législateurs que les rapports des individus qui la composent et qu'on cherche à émanciper de toute autorité abusive; l'individualisme qui caractérise la pensée juridique de la période révolutionnaire occulte la question de la famille qui n'est pas abordée en tant qu'objet mais, de manière plus souvent indirecte, sous le seul angle des individus qui se trouvent en être aussi des membres. La famille comme communauté tend à disparaître, dans les lois et projets de Code civil, au profit d'une association d'individus libres et égaux.

La première remarque qui s'impose à cet égard est l'absence de débat de fond sur la question de l'autorité dans la famille; ainsi que le fait observer Bernard Schnapper, on peut dire que «le despotisme familial n'a pas connu sa nuit du 4 août» (15). C'est toujours par le biais de «points techniques" (16) que les droits et obligations du père et du mari ont été soumis à la critique. L'autorité paternelle sur les enfants majeurs est, certes, abolie en tant que figure de l'autorité absolue du monarque et, à cet égard, ça n'est pas un hasard si l'abolition est votée à la veille de la proclamation de la

(13) Cf. Francis Ronsin, Le contrat sentimental, débats sur le mariage, l'amour, le divorce, de l'Ancien Régime à la Restauration, Paris, Aubier, 1990, et, du même, Les divorciaires, 1992, qui montre combien les lois sur (ou contre) le divorce sont liées aux changements politiques, de 1792 à 1975.

(14) Jacques MulLIEZ, «Droit et morale conjugale : essai sur l'histoire des relations personnelles entre époux ", Revue historique n' 569, PUF, juillet-sept. 1987, p. 86.

(15) Cf. Bernard SCHNAPPER, «L'autorité domestique et les hommes politiques de la Révolution ", dans La famille, la loi, l'État..., op. cit., p. 222.

(16) Points «techniques " tels que la correction paternelle en 1790 , la liberté de tester du père en 1791 et 1793 et les conventions matrimoniales en 1793 et 1795. 
République, le 28 août 1792 (17). Mais cette abolition a lieu à l'occasion d'un débat qui porte en réalité sur les substitutions; et la rapidité même avec laquelle il y est répondu montre aussi que la question de l'autorité dans la famille est moins pensée à ce moment-là comme un problème politique et philosophique que comme une des conséquences de l'émancipation juridique des individus.

L'égalité de partage entre les enfants, instituée par la loi du 7 mars 1793 sur les successions répond, elle aussi, à la volonté d'introduire dans la famille les mêmes principes que dans la société politique (18). Par cette loi, le père se voit retirer le dernier de ses moyens de pression ou d'obéissance : celui d'exhéréder l'enfant récalcitrant, puisque toute faculté « de disposer de ses biens, soit à cause de mort, soit entre vifs, soit par donation contractuelle en ligne directe, est abolie » (19).

Quant à l'autorité maritale, elle est abolie dans les deux premiers projets de Code civil présentés par Cambacérès (20) en 1793 et 1794. L'administration des biens doit désormais revenir aux deux époux comme . "le mode le plus conforme à cette union intime " (21) qu'est le mariage et parce que le « principe d'égalité doit régler tous les actes de [l']organisation sociale » (22), explique Cambacérès, le 9 août 1793.

\section{«Il n'y a plus de familles proprement dites »}

«Peut-il être question de ces dispositions bizarres d'après lesquelles la famille subsistait dans un seul homme? Il n'y a plus de caste, plus de familles proprement dites; il ne s'agit plus de faire des tiges. » (23)

(17) La rapidité avec laquelle il y est répondu lajsse d'ailleurs penser que la mesure demandée " profitait d'un large consensus"; il devenait en effet intolérable qu'un citoyen entre 21 et 25 ans, mineur du point de vue du droit civil, fût encore sous la domination d'un autre, ne serait-ce que pour obtenir l'autorisation de se marier. Cf. Bernard SCHNAPPER, «L'autorité domestique et les hommes politiques de la Révolution ", dans La famille, la loi, l'État..., op. cit., p. 229.

(18) « Il y a bien eu une révolution [explique Pierre Murat] : celle qui consiste à avoir osé penser la transmission du patrimoine en termes d'égalité politique et de droit de l'individu, et non plus seulement comme la perpétuation d'une lignée dont le chef temporaire imposerait ses commandements par-delà même la mort. [...] Le père n'est plus le premier magistrat de sa famille, parce que la famille n'est plus le premier groupe social : l'individu et la cité passent désormais avant; en conséquence, les lois, et l'État, qui les fait respecter, prennent au père une part de son pouvoir. " (Pierre MURAT, « La puissance paternelle et la Révolution française... ", dans La famille, la loi, l'État..., op. cit., p. 403).

(19) Article de la loi du 7 mars, cité par Pierre Murat, ibid, p. 403.

(20) Cf. A. M. DE BERGH, Le comité de législation et le droit de la famille dans les deux premiers projets de Code civil, Paris, thèse, 1956, $211 \mathrm{p}$.

(21) CAMBACÉRÈS, « Rapport sur le premier projet de Code civil devant la Convention au nom du Comité de législation civile et criminelle ", le 9 août 1793, dans P.-A. FENET, Recueil complet des travaux préparatoires du Code civil, Paris, 1836, t. 1, p. 4.

(22) Ibid., p. 5.

(23) Prugon, Archives parlementaires, t. 24, p. 598 (cité par Pierre MURAT, «La puissance paternelle... ", dans La famille, la loi, l'État..., op. cit., p. 403). 
Cette observation émise par un député lors de la discussion sur l'égalité successorale est particulièrement juste : sous la double action émancipatrice et égalisatrice opérée par les législateurs, la famille n'existe plus en tant que corps constitué. Pour le droit révolutionnaire, il n'y a pas de familles mais de petites sociétés d'individus dont les liens de conjugalité ou de parenté ne sont pas spécifiques par rapport aux liens civils (24). Il y a, dans cette absorption de la famille par la société civile, une grande similitude avec l'absorption, par le modèle familial, de la société politique sous l'Ancien Régime. D'une communauté politique inégalitaire et socio-naturelle, on passe à une association d'individus construite sur des principes rationnels, égalitaires et libéraux. Dans ce passage, la confusion entre ordre familial et ordre politique est demeurée inchangée; d'une nature inégalitaire venant justifier l'ordonnancement inégalitaire des rapports sociaux entre les hommes, on est passé à une nature égalitaire permettant à son tour de justifier la construction de la société politique, égalitaire elle aussi.

\section{2 ... et la République, une grande famille}

Le 26 germinal an II, le Comité de salut public bannit de Paris, des villes maritimes et des places militaires les anciens nobles et les «étrangers des pays avec lesquels la République est en guerre". Dans les jours qui suivent, des centaines d'hommes et de femmes font parvenir des pétitions au Comité pour expliquer que malgré les apparences, la loi ne saurait les concerner. Ces suppliants sont d'anciens officiers de la noblesse, d'ex-secrétaires du roi, des réfugiés politiques des Pays-Bas, des juifs étrangers, mais aussi des veuves, des couples divorcés, et des enfants nés hors mariage.

Le texte du décret est peu prolixe sur les liens de famille. Comme dans bien d'autres cas, le gouvernement révolutionnaire se montre plus soucieux de purger la nation de ses ennemis, réels ou imaginaires, que de régler les problèmes domestiques afférents. Mais pourquoi, dans ce cas, les pétitionnaires s'en réfèrent-ils aussi souvent à leur statut matrimonial ou familial pour justifier du caractère exceptionnel de leur situation? Pour répondre à cette question, il faut examiner la manière dont les révolutionnaires ont, pendant cette première période qui va de 1789 à 1794, soigneusement lié les questions de citoyenneté et d'état familial des personnes. Au-delà du traitement de la famille comme société politique, il existe en effet une législation qui, en matière d'accès à la citoyenneté, fait du lien familial une preuve d'appartenance nationale : c'est notamment le cas de la législation sur la naturalisation des étrangers.

(24) Cf. Julien BONNECASE, La philosophie du Code Napoléon appliquée au droit de la famille. Ses destinées dans le droit civil contemporain, Paris, 1928 (2e éd.), p. 84. 


\section{Citoyenneté nationale et contrats individuels}

Un des premiers objectifs de l'Assemblée nationale est d'en finir avec la structure corporatiste héritée de l'Ancien Régime, tout en donnant aux individus la possibilité de ne plus se définir en fonction de leur appartenance à ces ordres abolis, mais de leur adhésion volontaire et libre à la nation. Toute collectivité, toute communauté susceptible de s'interposer entre l'État et l'individu sera jugée et traitée comme un corps étranger; c'est ainsi que les aristocrates et autres membres de «corporations ", regardés collectivement comme étrangers, peuvent individuellement être considérés comme des citoyens français (25). Les juifs se voient ainsi accorder la pleine citoyenneté à condition qu'ils abandonnent leurs particularités culturelles et leurs institutions (26); quant aux étrangers au sens littéral du terme, ils peuvent être admis aux droits du citoyen français s'ils embrassent totalement les principes de la Révolution (27).

Le lien entre citoyenneté et participation active s'accentue à mesure que le terme de citoyen devient un mode d'interpellation plus courant : celui-ci est utilisé non seulement pour s'opposer à "noble », mais également pour remplacer le titre de Monsieur ou Madame, ainsi que celui de « régnicole » qui jusque-là distinguait légalement les Français des étrangers (aubains). Les premières lois révolutionnaires sur la citoyenneté nationale usent encore d'expressions d'Ancien Régime, comme « réputés français ", ou " naturels " (28) tandis que d'autres se réfèrent plus simplement à «citoyen français» voire «citoyen». La Constitution de 1791 établit ainsi qui sera considéré comme citoyen français; celle de 1793 définit ceux qui seront admis à «l'exercice des droits de citoyen », tandis que celle de l'an III se référera aux "citoyens français » et à "l'exercice des droits de citoyen ». Les usages indifférenciés de l'un ou l'autre terme créent (ou laissent voir) une ambiguïté permanente entre l'idée d'une participation active à la communauté politique des citoyens et une appartenance assignée à la nouvelle nation. De plus, l'idée que la citoyenneté

(25) Mirabeau en est bien sûr la figure la plus connue.

(26) Cf. Gary KATES, «Jews into Frenchmen : Nationality and Representation in Revolutionary France », dans Ferenc FEHER (éd.), The French Revolution and the Birth of Modemity, Berkeley and Los Angeles, University of California Press, 1990; Robert BADINTER, Libres et Égaux. L'émancipation des Juifs, 1789-1791, Paris, Fayard, 1989; ainsi que Frances MAL1NO, A Jew in The French Revolution: The Life of Zalkind Hounwitz, Oxford, Blackwell, 1996.

(27) Les exemples les plus fameux sont ceux de l'Américain Thomas Paine et du Prussien Anacharsis Cloots qui tous deux devinrent députés à l'Assemblée nationale.

(28) Ainsi, de ce décret de mai 1790 se référant à tous ceux « réputés français et admis, en prêtant le serment civique à l'exercice des droits de citoyen "; "déclarés naturels français ", ils « jouiront des droits attachés à cette qualité »; et de ce décret de décembre 1790 proclamant que les protestants ayant fui les persécutions et retournant dans leur pays seraient déclarés français naturels et reconduits dans tous les droits attachés à cette qualité. 
française doit être active, libre et fondée sur un contrat individuel est une constante des lois sur la citoyenneté (29).

À mesure que le processus révolutionnaire se déroule, l'État cherche à séparer ceux à qui la loi doit simplement protection, de ceux capables de participer directement au Souverain et de créer de nouvelles lois. Mais parallèlement à ce mouvement, la recrudescence de la violence rend de plus en plus abscons ce partage entre une participation active et une position purement passive et apolitique : tous les citoyens ne peuvent voter, certes; mais ils peuvent tous trahir la République, ne serait-ce qu'en quittant le territoire. N'est-ce pas ce qui est reproché à la jeune émigrée suppliant le gouvernement de ne pas la tenir pour responsable d'un départ qu'elle ne doit qu'à son obéissance conjugale et filiale? Les femmes sans-culotte persistent à clamer qu'elles font partie du souverain alors qu'à partir de 1793, elles sont officiellement exclues de toute vie politique (30). Même ainsi reléguées aux frontières de la cité, elles n'en conservent pas moins la pleine responsabilité de leurs actes en tant que citoyennes, puisqu'elles peuvent être reconnues coupables de crimes relevant de cette activité. Bien plus, il leur est demandé de prouver leur patriotisme et de faire allégeance à la nation révolutionnaire, en choisissant entre leurs liens familiaux et leur appartenance nationale. À ceux qui, invoquant l'indulgence des législateurs pour les épouses fidèles ayant respecté l'ancienne loi «sacrée, impérissable comme la nature dont elle émane, je veux dire l'autorité paternelle et maritale", le Bureau des lois, presque systématiquement, répond que «les lois sur l'émigration ont placé les devoirs envers la patrie avant tous les autres devoirs» et qu'«une femme invoquerait vainement pour légitimer son absence, tels liens qui l'attachent à son époux émigré, un enfant n'assegue-

(29) Selon les textes constitutionnels, ces conditions peuvent inclure un serment civique, une résidence en France, le mariage avec une Française, la nécessité d'avoir établi une entreprise commerciale ou agricole, de nourrir un vieillard ou de payer une contribution et enfin l'adoption d'un enfant. Cf. les Constitutions de 1791, 1793 et 1795 reproduites dans La nationalité française. Textes et documents, Paris, La documentation française, 1985. Pierre Rosanvallon caractérise ces liens comme passifs (résidence), politiques (serment) et actifs (sphère familiale et économique). La résidence n'est peut-être pas si passive si l'on prend en compte les lois révolutionnaires contre l'émigration. (cf. Pierre RoSANVALLON, Le sacre du citoyen..., op. cit., p. 73). Il faut noter également qu'il y eut bien des débats pour décider si de telles conditions s'appliquaient spécifiquement aux droits politiques ou à la citoyenneté en général, principalement à la fin des années 1790 et au début du XIX'c siècle (cf. Rogers BRUBAKER, Citizenship and Nationhood in France and Germany, Cambridge, Cambridge University Press, 1992; Pierre RosanVAllon, Le sacre du citoyen..., op. cit.; ainsi que Jean LECA, "La citoyenneté entre la nation et la société civile», dans Dominique COLAS, Claude EMERI et Jacques ZYLBERBERG (dir.), Citoyenneté et Nationalité. Perspectives en France et au Québec, Paris, PUF, 1991, pp. 479-505).

(30) Cf. Joan LANDES, Women and the Public sphere..., op. cit.; Dominique GodINEAU, Citoyennes Tricoteuses : Les femmes du peuple à Paris pendant la Révolution française, Aix-en-Provence, Alinea, 1988; William SEWELL, «Le citoyen/la citoyenne : Activity, Passivity, and the Revolutionary Concept of Citizenship ", dans Colin LuCAS (éd.), The French Revolution and the Creation of Modern Political Culture, Oxford, Pergamon Press, 1987, pp. 105-123; ainsi que Olwen HuFTON, Women and the Limits of Citizenship in the French Revolution, Toronto, University of Toronto Press, 1992. 
roit pas avec plus de succès les devoirs sacrés de la piété filiale...» (31). Quelques années devront passer pour que le gouvernement songe à éclaircir ce dilemme des épouses prises entre des devoirs aussi contradictoires.

\section{Les entrelacements de la famille et de la citoyenneté}

On a vu que les nouvelles lois sur la famille et la citoyenneté nationale partageaient des concepts communs, tels que la liberté individuelle et l'égalité ainsi que l'adhésion volontaire à la société. Mais les nouvelles lois sur la citoyenneté et la famille s'entrelacent d'une manière encore plus directe dans le champ de la naturalisation des étrangers et de leur accès à la citoyenneté.

Dans la France pré-révolutionnaire, le roi, considéré comme le père de son peuple, traitait de manière conjointe la légitimation et la naturalisation; et bien que l'Assemblée législative ait aboli le droit d'aubaine en 1791, l'État s'est approprié bien des aspects de cet ancien modèle, assimilant appartenances nationale et familiale. Ainsi, les législateurs utilisent-ils le terme d' «adoption » pour décrire le processus d'incorporation des étrangers (32); les pétitionnaires réagissant au décret de germinal an II usent du même langage en s'identifiant à des "enfants adoptifs de la République » (33). Enfin, le gouvernement adopte officiellement nombre d'enfants français après l'exécution de Louis XVI. La plupart étant orphelins, l'État promet d'agir en lieu et place de leurs parents biologiques. L'adoption de Suzanne Lepeletier, évoquée en introduction, n'est que la première d'une longue série. En juin 1793, Michel Azéma, dans un rapport sur l'adoption, proclame que «la patrie doit, à tous égards, tenir lieu de père à ceux dont les pères sont morts pour son service» (34) et que les enfants adoptifs de la patrie, puisqu' «il est incontestable que ces orphelins sont citoyens, appartiennent à la cité en naissant » (35). En juillet, un décret donne aux enfants abandonnés le titre d'«enfants naturels de la patrie "; et le 15 brumaire an II, la Convention adopte officiellement de jeunes enfants dont les parents se sont vu confisquer leur propriété par l'État. Mais à mesure que la Révolution déplace le cœur de la souveraineté - la localisant dans le corps social du peuple français plutôt que dans la personne du monarque - les relations familiales avec des citoyens français

(31) A.N., F7 4324, 3e série, dossier 93.

(32) Il est révélateur à cet égard que parmi les suppliants des pétitions concernant le bannissement des nobles et "étrangers " de Paris ( 26 germinal an II, cf. supra) on en trouve un certain nombre qui se présentent comme des « enfants adoptés » de la nouvelle République et en appellent à l'État pour qu'il les reconnaisse comme sa progéniture.

(33) A.N., D III 375, dossier Fontanelle.

(34) Michel AzÉmA, Convention nationale. Rapport et projet de loi Sur l'adoption présentés à la Convention nationale, au nom du comité de législation, par Michel Azéma, député du département de l'Aude, le 4 juin 1793, Paris, Imprimerie nationale, s.a., dans A.N., AD II 30.

(35) A.N, AD II 34. 
deviennent un des moyens pour les étrangers de devenir membres de la "grande famille» de la nation. Par ces lois, les législateurs suggèrent que les liens familiaux peuvent unir les individus, non seulement entre eux, mais également à la communauté politique (36).

À mesure que les législateurs révolutionnaires redéfinissent les relations familiales et citoyennes en termes de contrats individuels, ils posent les conditions de conflits potentiels entre la famille et la patrie. Toutefois, aucun des gouvernements de ces premières années de la Révolution ne paraît prendre conscience de ces tensions susceptibles d'émerger - probablement parce que de tels conflits avaient rarement éclaté sous l'Ancien Régime (37); ils allaient cependant découvrir que tel n'était plus le cas.

\section{La famille, « motif de la société civile et politique » (38)}

En 1797, les lecteurs du Journal d'économie publique se voient rendre compte, en des termes plutôt sarcastiques, d'un ouvrage portant sur «la famille considérée comme l'élément des sociétés » (39). Cet ouvrage est signé de Toussaint Guiraudet, tandis que le compte rendu est le fait du propriétaire du journal, Pierre-Louis Roederer. Le premier, un ancien député de l'Assemblée constituante qui fut proche de Condorcet et Mirabeau, est alors secrétaire général du ministère des Relations extérieures (40); quant à Roederer, c'est un personnage bien connu à l'époque, acteur de premier plan des premiers temps de la Révolution (41), propriétaire de la moitié du Journal de Paris, et fondateur du Journal d'économie publique. Les sarcasmes de Roederer sont dirigés contre les évidences proférées, selon lui, par Toussaint Guiraudet; selon l'auteur, l'époque serait venue d'abandonner le principe qui a établi que la nation n'était qu'une «agrégation d'individus",

(36) Ainsi, la Constitution de 1791 fait-elle du mariage avec une Française un moyen pour un étranger de devenir français, et l'acte constitutionnel de juin 1793 des soins apportés à un Français un moyen de devenir citoyen français. Enfin, les prêtres et les nonnes, hommes et femmes au statut particulier, peuvent prouver leur citoyenneté en se mariant (Roderick PHILLIPS, «The Attack on Celibacy in EighteenthCentury France ", dans Proceedings of the Annual Meeting of the Western Society for French Histony, 17, 1990, pp. 165-171).

(37) Sous l'Ancien Régime, hommes et femmes ne pouvaient être tenus de choisir entre lien national et lien matrimonial parce qu'il était impossible de rompre ce dernier, sauf par la mort de l'un des époux. Même la perte complète des droits de citoyenneté et de famille, par la mort civile, ne mettait pas fin légalement à leur union.

(38) L'expression est de Pierre-Louis RoEDERER, «Cours d'organisation sociale fait au Lycée en 1793 », dans CEuves du comte P.-L. Roederer, op. cit., p. 159.

(39) ROEDERER, « Réflexions sur l'ouvrage du citoyen Guiraudet, intitulé : De la famille, considérée comme l'élément des sociétés », Joumal d'économie publique, 20 thermidor an V (7 août 1797).

(40) Il est également l'auteur d'un grand nombre d'ouvrages ou fascicules portant sur des sujets très divers, tels que la doctrine de l'impôt, la garde nationale, les forges de la Côte d'Or, la nation, la langue politique et enfin, l'égalité.

(41) Cf. Thierry LENTZ, Roederer, 1754-1835, Metz, éd. Serpenoise, 1989. 
au profit d'une vision considérant celle-ci comme «un composé de familles »; c'est aux seuls chefs de famille qu'il faut accorder le titre de citoyen actif ou d'électeur; et c'est la puissance domestique qu'il faut accroître, afin de «n'avoir pas un roi pour la France» (42). Or, lui répond Roederer, il n'y a jamais eu d'individu tel que Guiraudet l'entend [ « jamais cette doctrine n'a existé, que je sache » (43)], mais seulement des chefs de famille ( il me paraît universellement reconnu, il l'est surtout en France, que les chefs de famille seuls sont citoyens »). Aussi, le retour auquel il aspire n'at-il pas lieu d'être, pour la bonne raison que le paterfamilias ne s'est en réalité jamais effacé au profit de « l'être idéal », « l'atome » décrits par Guiraudet.

Lintérêt de ce pseudo dialogue réside dans le décalage entre ces deux perceptions de l'individu citoyen. Pour l'un, l'époque est à un retour au pouvoir du père dans la famille pour restaurer l'autorité "civile", siège et condition de l'ordre dans la société démocratique (il s'agit donc d'un pouvoir politique du père de famille); pour l'autre, il n'y a rien à changer, le citoyen ayant toujours été ce paterfamilias requis par le premier, et la société politique ayant depuis le début de la Révolution fonctionné sur la base de cette autorité déléguée au chef de la communauté familiale considérée comme société politique. Il faut retenir de ce désaccord l'extrême ambiguïté dans laquelle a flotté la notion d'individu depuis le début de la Révolution. Nulle loi n'ayant pris la peine de préciser que seuls les paterfamilias étaient membres du Souverain; certains textes, certaines pratiques ayant au contraire pu laisser penser que toutes les personnes majeures, sans exception, appartenaient à cette communauté politique nationale; il n'est pas surprenant que l'individu-citoyen ait pu être assimilé par Guiraudet à cette unité élémentaire, cette fraction du corps social qu'est l'homme "physique ». Mais l'élu de l'Assemblée constituante oublie visiblement que, de fait, seuls les hommes majeurs non domestiques, c'est-à-dire les pères de famille au sens romain du terme (44), ont eu le droit de suffrage depuis 1789; et qu'il n'a pas été besoin de le préciser, justement, pour que l'ensemble des lois réglant les droits de cité marquent une cohérence certaine à cet égard. Il y a bien eu un consentement tacite à considérer le citoyen comme un paterfamilias en même temps qu'il y a eu des usages du terme « individu » ayant pu provoquer certains malentendus.

(42) Charles-Philippe-Toussaint GuIRAUDET, De la famille, considérée comme l'élément des sociétés, Paris, 1797; voir en particulier les pages 193 à 200.

(43) ROEDERER, « Réflexions sur l'ouvrage du citoyen Guiraudet... », op. cit., p. 98.

(44) C'est Roederer qui donne cette définition du citoyen sous la Révolution : «Bien entendu que sous ce mot, comme chez les Romains, sous le mot de pater familias, on doit comprendre non seulement le père de famille, mais aussi celui qui peut l'être. C'est en vertu de ce principe que les femmes, les mineurs, les domestiques, les soldats mêmes, sont exclus des droits de cité " (ROEDERER, « Réflexions sur l'ouvrage du citoyen Guiraudet... ", op. cit., p. 98). 
Ces malentendus sont évidemment pleins d'enseignements; ils expliquent en partie pourquoi la Révolution a tant tardé à distinguer société domestique et société politique; en 1797, les textes de Guiraudet et Roederer montrent que si malentendu il peut y avoir sur ce qu'a été l'unité élémentaire de la société politique, il y a $a$ contrario un accord définitif sur ce qu'elle doit être désormais, à savoir : la famille incarnée par son chef naturel, le citoyen. Or, bien d'autres textes témoignent de cet accord sur le caractère «familialiste» à donner aux lois qui règlent l'appartenance nationale et la participation électorale; accord qui, à en croire Roederer, ne serait rien de plus qu'une prise de conscience de ce qui a été, simplement. Il est vrai que sa position est remarquablement cohérente. Mais il est vrai aussi que bien des hommes, à l'instar de Guiraudet ont, malgré une législation globalement construite sur le paterfamilias, pensé œuvrer pour et par l'homme élémentaire et non pour et par le chef de famille. Toujours est-il qu'au lendemain de la Terreur, après bien des années de conflits nés de notions par trop ambiguës, les législateurs conviennent de la nécessité de rétablir le père dans ses droits, et la famille dans sa spécificité; et de faire de celle-ci, non plus un modèle, mais un «motif » de la société civile et politique.

\subsection{Vers un début de résolution des conflits}

\section{Les émigrées et la paix sociale}

En 1797, les défenseurs des émigrés commencent à en appeler à une amnistie pour les femmes et les enfants qui ont quitté la France.

Pour ce faire, la plupart utilisent des arguments fort anciens, comme ceux de notre jeune émigrée arguant de l'insignifiance politique des femmes et de l'incapacité (voire du peu de désir) des dépendants à choisir entre liens familiaux et nationaux. De telles réclamations apparaissent avec une fréquence régulière aux lendemains de Thermidor. Lally-Tolendal, un exparlementaire, membre important de l'Assemblée nationale de 1789 , prend la tête de ce mouvement en faveur d'un retour des émigrés en France et de leur rétablissement dans leurs droits de citoyens français. Sa Défense des émigrés français connaît quatre éditions durant la seule année 1797, sans compter les nombreuses autres qui se succéderont sous la Restauration (45). Bien des arguments de Lally-Tolendal sont déjà répandus parmi les défenseurs des émigrés; mais tout en appelant à une amnistie générale, lui insiste sur le cas des femmes et des enfants qui, selon lui, doivent être traités séparément des autres. L'ex-parlementaire justifie une telle exception en arguant

(45) Cf. Trophime-Gérard DE LALLY-TOLENDAL, Défense des émigrés français, adressée au peuple français, Paris, Libraires et marchands de Nouveautés, an V (1797). Parmi les autres éditions, deux furent publiées à Paris (Cocheris, an VI, [1797] et s.l. [1797]) et une à Hambourg (P. F. Fauche, 1797). Les pages référencées ici sont extraites de l'édition des Libraires et Marchands de nouveautés. 
que les femmes forment un groupe à part dont les relations avec la nation française sont intrinsèquement différentes de celles des hommes. Le gouvernement, selon lui, ne saurait placer le devoir patriotique au-dessus de la loyauté à la famille, puisque «la patrie est née de la famille, [et que] la nature a précédé la cité » (46).

On retrouve le même genre d'arguments chez l'un des protagonistes du débat, Leuliete :

«Il est cruel, sans doute, de fermer irrévocablement les barrières de la patrie à tant de femmes, dont le crime fut un attachement trop religieux aux opinions des mortels chéris, à qui des nœuds sacrés avaient uni leur sort. » (47)

Justement parce que leur loyauté à la famille a précédé leur loyauté à l'État, de telles femmes ne souhaiteront pas rentrer en France sans leurs époux; elles ne devraient d'ailleurs même pas y être autorisées. Leuliete comprend l'idée d'une amnistie partielle comme destructrice de l'unité familiale, car :

"quels charmes pourrait avoir, pour une femme sensible, une terre dont son époux serait éternellement banni... ce serait une grâce illusoire; ce serait méconnaître les plus nobles sentiments de la nature, que de rappeler l'épouse et de fermer la porte à l'époux, de rappeler le père et d'éterniser la proscription du fils. » (48)

La double idée selon laquelle la famille devrait, en ce qui concerne les femmes, être prioritaire sur les devoirs patriotiques et les femmes n'avoir pas les devoirs de citoyennes incombant aux hommes semble trouver sa réalisation lorsque Napoléon déclare une amnistie partielle des émigrés, le 28 vendémiaire an IX (20 octobre 1800). L'article 5, tout spécialement destiné au cas des femmes, accorde en effet une amnistie non seulement à celles qui ont quitté la France sous l'autorité de leur époux, mais aussi à celles qui ont émigré de leur propre chef, indépendamment des charges d'émigration pouvant peser sur leur époux.

\section{Les femmes, toujours citoyennes?}

Les pratiques électorales, ainsi que les uságes qui ont été faits des règlements électoraux pendant la période révolutionnaire montrent que la répartition politique des personnes selon leur situation familiale reste très ancrée, même si, en abandonnant les distinctions honorifiques de la propriété au profit de la capacité politique qu'elle désigne, les législateurs vont peu à peu introduire des modifications conséquentes.

(46) LALly-Tolendal, Défense des émigrés, p. 35, p. 29.

(47) Jean-Jacques LEULlETE, Des émigrés français, ou réponse à $M$ de Lally-Tolendal, Paris, Cercle-social, 1797, p. 19.

(48) LeULIETE, Des émigrés français..., op. cit., pp. 20, 23. 
Une liste d'habitants de la commune de Baron est très significative à cet égard de la manière dont on répartit les personnes. Cette liste d'habitants nous est donnée par Serge Aberdam, parmi toute une série d'autres textes montrant la très large participation des femmes aux assemblées électorales pendant la période révolutionnaire, et l'année 1793 en particulier (49). Elle est, nous dit-il, «disposée en trois colonnes qu'un rédacteur a intitulées : Hommes, Femmes, Enfants ». Or, sous ces trois appellations, celui-ci a défini comme Hommes tous les chefs de famille, y compris les veuves; comme Femmes, les épouses et filles, mais également les domestiques dont certains "semblent être en réalité des hommes »; et comme Enfants, tous ceux qui ont jusqu'à 24 ans. D'autres textes mis à jour par Serge Aberdam vont dans le même sens (50); mais celui-ci est le plus caractéristique de cette représentation qui consiste à situer les personnes selon des capacités politiques directement attachées à la propriété, et non selon une appartenance sexuelle qui, même en 1793, ne s'avère pas pertinente en la matière, du moins d'une manière généralisée. Hommes et Femmes sont des catégories sociales d'Ancien Régime qui renvoient à une administration des biens familiaux où l'appartenance sexuelle n'induit d'empêchement que pour les femmes subordonnées. Les législateurs modifient implicitement ces catégories en introduisant, à l'intérieur des familles, la féminité comme critère d'incapacité à représenter la nation, excluant les veuves propriétaires de toute participation directe à la souveraineté. Mais ils n'iront pas au «bout» de cette logique, puisqu'ils ne feront pas de l'appartenance sexuelle un critère de distinction politique traversant, au-delà de la famille, toute la société : on sait que les domestiques, ces autres membres de la famille, demeurent exclus du droit électoral pendant toute la période révolutionnaire (51).

(49) Je renvoie au chapitre passionnant consacré à la participation électorale des femmes pendant la période révolutionnaire par Serge ABERDAM, L'élargissement du droit de vote entre 1792 et 1795 au travers du dénombrement du comité de division et des votes populaires sur les Constitutions de 1793 et 1795, thèse d'histoire sous la dir. de Michel Vovelle, Paris 1, Sorbonne, 2001, p. 19 (qui donne comme référence pour cette liste : AD de l'Oise, liasse cotée provisoirement 2 Lp 9048; Baron, chef-lieu de Canton, réponses au questionnaire de l'été 1793). Les textes qu'il nous livre sont d'un intérêt immense pour la connaissance de la situation politique des femmes.

(50) Ainsi de cette liste adressée aux « autorités » des 196 « citoyens pères de famille de la commune de Crouttes », et qui comprend 24 veuves. AD de l'Orne, L 593-595; district d'Argentan. Cité par Serge ABERDAM, op. cit., p. 18.

(51) La seule Constitution qui les aie jamais inclus, est celle, caduque, de 1793 ; encore le fait-elle d'une manière tout à fait particulière, puisqu'en imposant une condition de domicile à l'exercice de ce droit, elle continue en fait de les exclure. Non seulement ils ne sont pas admis à l'exercice des droits du citoyen, mais à l'instar des femmes et des enfants, il n'est pratiquement jamais un député pour soulever la question de leurs droits civiques.

Cette particularité de la Constitution passe en général inaperçue : la condition de domicile n'est en effet pas mentionnée, dans la plupart des histoires du suffrage, comme particulièrement restrictive à l'endroit des domestiques. Or, c'est pourtant bien dans cet esprit que les législateurs ont réintroduit ce critère, comme en témoigne ce passage des débats au cours duquel Thuriot obtient la modification du projet initial dans lequel était employé le terme "réside": "Je pense qu'il faut déterminer l'état de l'individu, car un homme riche pourrait occuper un grand nombre d'ouvriers ou de domestiques pour voter en sa faveur, et 
Au même moment, un débat a lieu à la Convention sur les droits politiques des femmes (dans le cadre du projet de Constitution de 1793); il se solde par la défaite des partisans de l'égalité entre les individus de l'un et l'autre sexe, minoritaires au sein des Girondins (52) et montre, à travers le projet de Robespierre, «l'hostilité de la direction montagnarde à une extension du droit de vote aux femmes » (53), hostilité largement majoritaire à la Convention. Serge Aberdam fait l'hypothèse que, jointe à l'édiction de la loi sur le partage des communaux, la publicité des débats à la Convention sur le droit de vote des femmes a pu créer une certaine confusion «en matière d'admission des femmes au droit de vote en juin-juillet 1793 » (p. 69), confusion qui aurait ouvert la voie à des pratiques d'élargissement du suffrage aux femmes et de revendications en faveur de leur inclusion au souverain. Toujours est-il qu'il relève un grand nombre d'actes d'adhésion à la Constitution auxquels ont participé les femmes pendant l'été 1793, tout en soulignant l'écart entre cette participation et les procès-verbaux envoyés à la Convention qui n'en font que rarement mention. «[...] tout se passe comme si, lorsque la Convention propose le vote de la Constitution comme un moyen d'affirmer l'unanimité nationale en face des périls, une partie des femmes en vient à considérer qu'il y a là une place qui leur revient, par droit ou par fonction, par antériorité ou par innovation : comme veuve domiciliée, comme épouse intéressée au partage ou au non-partage des communaux, comme contribuable, comme mère de volontaire, comme sœur de requis, comme épouse de marin, comme travailleuse aux fournitures, mais peut-être aussi comme personne humaine »(54). De même, bien des femmes parmi les contestataires du décret de germinal an II sur le bannissement des étrangers ont mis l'accent sur leur patriotisme, arguant de leur divorce pour prouver leur adhésion volontaire à la nation (55).

Ce sont les années suivantes, c'est-à-dire postérieures à Thermidor, qui semblent marquer, sinon une réelle rupture, du moins l'infléchissement le plus net dans la prise en considération des femmes comme partie du

vous devez prévenir cet abus. Je demande qu'on substitue au mot réside le mot domicile; car pour être domicilié, il faut avoir loué l'appartement ou avoir acheté la maison où l'on loge " (THURIOT, Archives partementaires, 11 juin 1793, t. LXIV, p. 283, cité par Bertrand HÉRISSON, Lévolution de la citoyenneté en droit public français, thèse de droit public sous la dir. de E. Picard, Paris 1, 1995, [exemplaire dactylogr., 417 p.], p. 237). Cf. Anne VerJus, Les femmes, épouses et mères de citoyens..., op. cit., chap. 1 et chap. 3.

(52) Voir le récit très précis qu'en fait Serge Aberdam dans sa thèse, pp. 38-41.

(53) Ibid., p. 42.

(54) Serge ABERDAM, op. cit., pp. 110-111.

(55) Ainsi de la citoyenne Bouillard qui demande à ce que le divorce, en tant qu'acte volontaire, rupture de contrat, ne soit pas assimilé à un "simple veuvage " : « Je te demande encore si mon divorce ne m'a pas ôté cette tache de noblesse que me donnait mon mariage avec un noble. Il me semble qu'il doit avoir cette vertu, puisqu'en rompant le lien conjugal, il m'a depouillée de tous mes avantages matrimoniaux. comment se pourrait-il qu'il laissât subsister les titres ou les qualités ci-devant honorifiques? On ne peut comparer le divorce sous tous les rapports avec la mort naturelle du mari. " A.N., D III 373. Ce point est développé dans le chapitre 2 de Jennifer HEUER, Foreigners, families..., op. cit. 
Souverain. Car au-delà du cas des émigrées, on trouve dans ces années-là d'autres témoignages significatifs de l'abandon relatif de la reconnaissance du lien politique entre les femmes et la nation (56). L'un des plus explicites est rapporté par Roederer dans son Journal de Paris, en juin 1796. Alors qu'il conteste, lors d'une conversation dans un café, l'usage " revenu fort à la mode » au théâtre du terme de "Monsieur » pour dire citoyen, et que son interlocuteur argue de l'usage encore plus répandu du terme de «Madame ", Roederer répond :

«Ceci, répartis-je, est autre chose. Les citoyens, dans la République française, sont les membres de l'État. Être membre de l'État, c'est avoir un droit politique. Le titre de citoyen est donc un titre politique. Mais une femme n'est que membre de la famille. Elle n'a aucun droit politique dans l'État. Elle ne doit donc porter aucun titre politique. » (57)

Roederer explique alors à son adversaire que «citoyenne» ne peut pas être le féminin de citoyen, comme «présidente » l'était de "président » sous l'Ancien Régime :

«C'est qu'autrefois les emplois publics s'obtenaient toujours par faveur ou par argent, c'était madame qui les obtenait, point monsieur, madame en était aussi capable que monsieur, et souvent les exerçait au moins par moitié avec monsieur. Mais dans les professions où il fallait payer de sa personne, comme celles de jurisconsulte et de médecin, le titre du mari ne passait point à la femme. Or, le devoir de citoyen, est de ceux pour lesquels il faut payer de sa personne.» (58)

On retrouve une idée parfaitement similaire, une année plus tard, lors du procès Hoppé-Lange; par la confrontation entre un père étranger et une mère française, tous deux non mariés, ce procès célèbre [où interviennent des avocats de renom, parmi lesquels Portalis (59)] a l'intérêt de mettre directement en opposition le principe de la puissance paternelle et celui des droits attachés à l'appartenance nationale (60). Or, à Portalis qui, pour la défense des droits du père, remarque que «l'avantage politique de vivre sous un gouvernement où chaque citoyen est une portion du souverain " [...] est « nul pour un sexe qui n'y participe pas» (61), l'avocat de la citoyenne Lange, Duveyrier, tout en reconnaissant que la participation des femmes au Souverain n'est certes qu'indirecte, répond qu'elles y participent néanmoins

(56) Cf. Jennifer HEUER, Foreigners, families..., op. cit., chap. 4, notamment le cas Hoppé-Lange.

(57) Roederer et CoranceZ, "Aux auteurs du Joumal de Paris ", Journal de Paris, n* 19 juin 1796.

(58) Ibid.

(59) Les autres défenseurs de Hoppé sont Tronson du Coudray, Muraire et Cambacérès

(60) Cf. Jennifer HeUer, Foreigners, families..., op. cit., chap. 4.

(61) PORTALIS et al., Consultation pour M. Hoppé contre la citoyenne Lange, sur une question d'éducation, Paris, Imprimerie nationale, an V (1797), p. 19. 
" par leurs époux, par leurs enfants, par leurs fils, par tous les avantages que la nature du contrat-social a mis en communauté » (62).

On passe à partir de la seconde partie de la Révolution à un système politique où posséder devient moins important qu'être reconnu capable de posséder; et où même la hiérarchie familiale tient moins compte du rapport à la propriété que des «différences naturelles» entre les êtres (les veuves n'étant plus, politiquement, des chefs de famille mais d'abord des membres féminins de la famille, à ce titre susceptibles de trouver dans celle-ci un homme pour faire représenter leur propriété).

\subsection{Formaliser les implicites : "les chefs de famille seuls sont citoyens»}

On a vu que la volonté des femmes de signifier leur appartenance au Souverain, très affirmée jusqu'en 1793, s'était heurtée aux hésitations des fonctionnaires tenus de normaliser ces pratiques en ne décomptant que les votes masculins. Le témoignage de Roederer, ainsi que le procès opposant ce père étranger et cette mère française, ont montré comment, de l'appartenance éventuellement participative à la nation souveraine qui pouvait être défendue dans les années 1789-1793, les femmes sont insensiblement en train de passer à une appartenance protectrice, porteuse de droits certes, mais par l'intermédiaire quasi exclusif des citoyens auxquels elles sont liées (père, époux, fils). C'est à partir de la seconde partie de la Révolution, postérieure à la Terreur, que tout semble changer; et de fait, tout change si l'on s'en réfère aux textes des lois électorales de cette période.

Le citoyen et les membres de la famille

Aux lendemains de la Terreur, les législateurs veulent en finir avec les pratiques déviantes, mal contrôlées et prêtant à cette confusion génératrice de pétitions, de procès et de comportements déviants : il s'agit de parvenir à un «art social " achevé, de fermer les ouvertures en définissant plus strictement les notions, les droits des uns et des autres, au premier rang desquels le droit de voter et la situation dans le corps politique.

Tout change à partir des lois électorales de l'an X (1802) : le citoyen n'y est définitivement plus le propriétaire réel (63); il suffit qu'il soit situé dans un ensemble qui le désigne comme apte à voter, pour que, même sans propriété

(62) H. DUVEYRIER, Réponse à la Consultation. Faite par M. Hoppé Hambourgeois, et signée Portalis, Tronson du Coudray, Muraire, et Cambacérès, Paris, Renauderie, s.d.

(63) Si tant est qu'il l'ait jamais été : les recherches menées jusqu'à maintenant n'ont pas permis d'établir sur quelle base (en l'occurrence patrimoniale) l'équivalent de trois journées de travail par exemple, néces saire pendant une grande partie de la période révolutionnaire à l'exercice du droit de suffrage, était calculé. 
personnelle, il puisse devenir électeur, voire éligible. En effet, c'est d'une manière très explicite que, désormais (64), les lois électorales prévoient que :

«l'on "comptera au mari les contributions de toute nature payées par sa femme, quoique non commune en biens" (art. 66); "au père les contributions payées sur les biens de ses enfants mineurs" (art. 67); qu'un "citoyen dont le père paie une somme totale de contributions assez forte pour être des six cents plus imposés de son département pourra, si son père consent par une déclaration authentique, visée du maire du lieu de son domicile, être inscrit en sa place comme plus imposé sur la liste des éligibles" (art. 38); enfin, que "si une femme veuve et non remariée paie une somme de contributions assez forte pour être du nombre des six cents plus imposés, elle pourra désigner un de ses fils majeurs pour être inscrit sur la liste des éligibles comme plus imposé" [art. 69]. » (65)

Le droit électoral va dès lors considérer l'électeur comme s'il était l'héritier universel de la communauté des biens de toute la parenté, ascendante et descendante, du couple qu'il forme avec son épouse. C'est là une définition originale de la famille, dans la mesure où elle ne tient compte ni des propriétaires réels, ni même des éventuels futurs propriétaires : la délégation de contributions est une opération politique, sans conséquences en aval sur la transmission ultime de la propriété, quoique dépendante en amont de la mise en évidence d'un lien de parenté. Cette définition du suffrage que l'on peut dire patrimoniale met en avant une approche de la famille, tout à fait spécifique à la loi électorale puisqu'elle contredit les principes du Code civil en matière de droit successoral. Ce faisant, elle répond bien à la conception que les législateurs, depuis le Directoire, commencent à se faire de l'électeur comme «individu social», voire comme "père de famille » si l'on se souvient du dialogue entre Guiraudet et Roederer. La contradiction n'est pas surprenante en soi, puisqu'on a vu combien il devenait banal de constater, voire conseiller, aux lendemains de Thermidor en particulier, une saine distance entre sphères politique et domestique, toutes deux incarnées

(64) Désormais, et pour le demi-siècle qui suit, c'est-à-dire jusqu'à la veille de la Révolution de 1848, ce que l'historiographie méconnaît généralement. Nous nous permettons de renvoyer à la thèse d'Anne Verjus (1997) dont le chapitre 4 est essentiellement consacré à cette question des droits et possibilités conférés à l'électeur potentiel.

(65) Arrêté du 19 fructidor an X (6 septembre 1802), contenant règlement pour l'exécution du senatus-consulte du 16 thermidor an X, relativement aux assemblées de canton, aux collèges électoraux, etc. (Bull. CCXIII, $\mathrm{n}^{\circ}$ 1964, Mon. du 22 fructidor an X, dans la Collection complète des lois, décrets, ordonnances, règlements, et avis du conseil d'État, publiée sur les éditions officielles du Louvre, de l'imprimerie nationale et du bulletin des lois; de 1788 à 1824 inclusivement, par ordre chronologique, avec un choix d'instructions ministérielles, et des notes sur chaque loi..., par J.-B. DuVERGIER, t. 13, p. 291). À partir de cette date, les lois électorales suivantes continueront d'admettre de manière explicite l'inclusion, parmi les contributions comptées à l'électeur, de celles de son épouse, de ses parents et de ses beaux-parents; cf. A. VERJUS, « La veuve et son gendre dans la stratégie électoraliste libérale sous la monarchie de Juillet ", communication à la journée d'études sur le suffrage, organisée par la Société des études robespierristes, Paris, mars 2001 (à paraître). 
dans la complémentarité des membres du couple conjugal (66). Elle peut étonner malgré tout, par son contenu : ne voit-on pas la sphère politique par excellence, c'est-à-dire l'exercice des droits électoraux, être pensée en fonction d'une logique "familialiste", tandis que le Code civil, qui régit les rapports familiaux, répond, lui, à une logique individualiste ? C'est par l'entremêlement. Les deux sphères que se résout l'apparent paradoxe. Car, dans l'esprit des législateurs - aisément discernable dans les constructions juridiques qu'ils mettent en place comme dans les discours des plus prolixes d'entre eux -, c'est par la figure du citoyen-père de famille justement que se fait le lien entre les deux sphères; aussi, le citoyen peut-il être l'unité élémentaire dans la sphère électorale, tout en tenant ses capacités des personnes qui composent la sphère familiale.

Il faut admettre qu'une évolution semblable à celle qui caractérise l'appartenance nationale / familiale des femmes touche la définition des droits politiques attachés au statut de chef de famille. Alors qu'au regard du droit, il ne semble pas y avoir d'ambiguïté, il en va différemment dans la sphère des pratiques, laissées à l'interprétation par un trop grand flou des notions, des catégories utilisées par les législateurs, peu préparés sans doute aux «détournements» qui seront faits de la loi. C'est ainsi que les chefs de famille sont assimilés dans un premier temps (qui va au moins jusqu'en 1793) à un statut social renvoyant strictement à la situation familiale (chef du foyer) des personnes; et que ça n'est que dans un second temps, lorsque l'application des textes de lois électorales s'est diffusée, normalisée, qu'on exclut de l'usage de ces droits statutaires celles qui n'en ont pas les vertus politiques présumées, à savoir : les veuves et demoiselles propriétaires. La sphère politique est une invention qui, comme l'a noté Patrice Gueniffey pour ce qui concerne les pratiques électorales (67), tarde à s'imposer; dans le domaine qui nous occupe, on voit bien que les droits du citoyen sont, d'emblée, destinés au seul paterfamilias, sur la base des anciens droits électoraux qui, interdisant aux femmes propriétaires d'être présentes dans les

(66) On peut encore ajouter, au détour de ce rappel, une intervention que Roederer insère en guise de conclusion d'un dialogue épique sur Voltaire et les femmes publié dans le Joumal de Paris, en 1796, et où il met en garde ses contemporains contre un éventuel retour au règne des distinctions féminines; la modestie et l'obscurité, y dit-il en substance, sont le devoir de toutes les femmes : «Dans une république, où toutes les naissances sont égales; où les lois, sans détruire l'inégalité des fortunes, doivent tendre toutes à empêcher les fortunes excessives; où l'amour du travail doit être l'esprit général parmi les hommes, la modestie et l'obscurité sont le devoir de toutes les femmes. La République française est perdue si les femmes peuvent y prétendre à des distinctions. Si elles en obtiennent par l'esprit, elles en obtiendront bientôt par la figure. Si elles peuvent obtenir des distinctions, bientôt elles auront une autorité, et leur empire dans l'empire sera infailliblement contre lui, parce que, ne pouvant le partager, la nature leur dira de le combattre» (ROEDERER, Joumal de Paris, 26 messidor an IV-14 juillet 1796).

(67) Cf. Patrice GUENIFFEY, Le nombre et la raison, La Révolution française et les élections, Paris, EHESS, 1993, 553 p.; ainsi que "Innovations et héritages dans les systèmes électoraux de la période révolutionnaire ", communication aux Journées "Antoine Bamave " d'Histoire constitutionnelle : Le Suffrage, citoyenneté, élections et démocratie (XV/e-XX' siècle), Università degli Studi di Macerata, 13-16 septembre 1995. 
assemblées électorales, les obligeaient à se faire représenter; mais on constate aussi que cette conception détachée des droits de la propriété au sens strict (puisqu'il n'est pas toujours nécessaire d'être propriétaire pour être électeur; et qu'il ne suffit pas d'être propriétaire non plus, dans le cas des veuves) s'est longtemps heurtée à des usages ruraux faisant primer ces droits de la propriété sur ceux de la personne.

Il y a un moment où les législateurs, au lieu d'opérer une simple réaction, c'est-à-dire un retour à l'ordre ancien suite aux errements et horreurs de la Terreur comme on l'a supposé (68), s'emparent aussi de questions qui sont sous-jacentes depuis le début. Autrement dit, il y a moins un passage de l'ordre individualiste à l'ordre "familialiste » qu'une évolution des conceptions du rapport entre famille et cité du point de vue de l'organisation politique. Ce ne sont pas tant les situations qui changent (même si elles changent à la marge) que les mots utilisés pour qualifier ces situations : en l'occurrence, les législateurs passent de l'individu au chef de famille, parce qu'ils réalisent que l'individu dont on négligeait de définir les contours était, depuis le début, un père de famille au sens romain de l'acception. Roederer, qui avait très tôt (1788) réfléchi au concept de citoyen, avait d'emblée fait le rapprochement; Guiraudet, personnage de moindre envergure (intellectuelle et sociale), ne le fait qu'en 1797; la majorité des membres de la Convention sont dans son cas plutôt que dans celui de Roederer : ils réalisent soudain que l'individu n'est pas la «personne humaine », mais le paterfamilias. Ils le disent, ils en voient l'intérêt, ils en font sans mal le fondement du droit; sans mal parce que de fait, les femmes et les enfants ont été exclus de la citoyenneté depuis le début.

C'est le paterfamilias, si bien décrit par Roederer, qui fait à lui seul le lien entre les deux univers. Il est à la fois l'individu de la république démocratique, et l'incarnation d'une autorité politique élémentaire reconnue, à partir du Directoire, nécessaire à son fonctionnement. La représentation en termes de sphères distinctes, autrement dit, est une figure paradigmatique d'un ensemble de lois qui, elles, sont généralement composites. C'est vrai des lois électorales, qui envisagent des unités à la fois individuelles et familiales (la famille étant l'unité politique incarnée par un seul). Mais c'est aussi vrai du Code civil, dont on va voir qu'il est tout autant construit selon des préceptes à la fois individualistes et familialistes.

\section{L'institution familiale et l'adoption nationale dans le Code civil}

En 1796, dans son troisième projet de Code civil, Cambacérès renonce à l'administration commune des biens dans le mariage et s'en justifie sans

(68) Cf. Suzanne DESAN, «Reconstituting the Social after the Terror : Family, Property and the Law in Popular Politics », Past and Present, 164, 1999. 
aménité ni regret apparent. Son projet ne sera pas retenu par le Conseil des Cinq Cents; mais l'administration commune, dans les projets suivants et jusqu'au Code civil, est définitivement abandonnée (69), au profit d'une administration rendue au mari, du respect à la nécessité de maintenir, dans la famille, une unité de direction, et à la «naturelle prééminence» de l'homme en cette matière (70).

Cela n'est pas tout. L'égalité des époux, d'abord entière pour ce qui concernait les motifs du divorce dans la loi de 1792, avait été entamée dès 1794 par le rétablissement, dans le premier projet de Code civil, d'une différence entre l'adultère commis par l'homme et celui commis par la femme : autant le mari pouvait toujours demander le divorce pour cause d'adultère de son épouse (71), autant celle-ci ne le pouvait désormais que si son époux avait tenu sa concubine dans la maison commune. C'est ainsi qu'un mari était considéré comme la «victime » de l'infidélité de sa femme alors que celle-ci ne pouvait se plaindre que d'être «délaissée » (72). Quant aux effets du divorce dans la loi de 1792, ils montrent déjà que le législateur tenait davantage à respecter la liberté des époux - liberté de dissoudre le contrat que leur égalité (73), puisque la femme est privée de tous ses droits et bénéfices dans la communauté de biens lorsque le divorce est obtenu contre elle (et notamment pour « dérèglement de mœurs » notoire) alors que l'inverse n'est pas vrai (74).

Vers l'an V, on commence également à remettre en cause le divorce par consentement mutuel. Les règles du Code civil de 1804, établies en fonction de l'individu, comme dans les premiers temps de la législation intermédiaire, demeurent un reflet de l'article 2 de la Déclaration des droits de l'homme et du citoyen de 1789 (75). De ce fait, le mariage y est encore et toujours défini comme un contrat civil, révocable de surcroît, liant entre elles deux personnes libres et égales. Mais, et c'est là que les distorsions apparaissent entre les deux philosophies contradictoires qui imprègnent le Code, dès que ces individus sont mariés, ils sont contraints de se soumettre à des rôles déterminés en fonction de leur appartenance sexuelle : c'est ainsi qu'ils deviennent des époux aux droits et devoirs différents, sans qu'il leur soit

(69) Cf. Nicole ARNAUD-DUC, «Le droit et les comportements, la genèse du titre V du livre III du Code civil : les régimes matrimoniaux ", dans La famille, la loi, l'État..., op. cit.

(70) On retrouve les mêmes arguments que ceux développés dans ces différents projets dans l'ouvrage d'un ancien avocat, Élie LUZAC, Du droit naturel, civil et politique, Amsterdam, 1802, t. I, pp. 268-269.

(71) Cf. « Le maître et l'infidèle. Des relations personnelles entre mari et femme de l'ancien droit au Code civil ", dans La famille, la loi, l'État..., op. cit., p. 439.

(72) Ibid.

(73) Cf. Jacques Mulliez, «Droit et morale conjugale : essai sur l'histoire des relations personnelles entre époux », op. cit., p. 88.

(74) Cf. Jean PORTEMER, « Le statut de la femme en France depuis la reformation des coutumes jusqu'à la rédaction du Code civil ", op. cit., p. 483.

(75) Cf. Julien BONNECASE, La philosophie du Code Napoléon..., op. cit., p. 64. 
possible de déroger à cette règle. Les principes d'égalité et de liberté ont définitivement disparu de l'organisation des rapports au sein du couple.

Le mariage n'est pas construit comme les contrats du droit commun mais s'apparente plus à une «institution ", telle que Julien Bonnecase l'a définie (76). Ainsi dans le mariage, le consentement des parties, public et solennel, s'inscrit-il dans un cadre bien déterminé dont les époux ne peuvent sortir ; l'officier de l'état-civil n'est pas là, comme dans le cas d'un contrat commun, pour constater les volontés mais pour déclarer les époux unis par le mariage (77). En outre, le mariage est public alors que le contrat est souvent privé. Le dol étant exclu, les consentements se trouvent moins protégés que dans le droit commun où il est le grand facteur d'annulation des contrats. Un mineur peut, avec l'autorisation de ses parents, se marier (art. 148) alors qu'il est incapable de figurer dans un contrat (art. 1398). Le divorce lui-même ne peut plus être considéré comme la marque de la contractualisation du mariage, alors que même s'il y a consentement mutuel des époux il est requis des faits précis pour les autoriser à se séparer (art. 1132) (78). Dans ce cas, le "consentement » ne sert plus qu'à cacher des causes de divorce qu'il convient de taire pour protéger l'honneur des familles (79) : on est loin de la loi de 1792 instituée au nom de la liberté des individus.

Enfin, et contrairement à l'article 686 qui prohibe l'établissement de droits sur la personne, «le mariage engendre à la fois, au profit et à l'encontre des époux, des droits respectifs sur la personne l'un de l'autre» (80). Ainsi, la femme doit-elle obéissance à son époux. Elle ne peut avoir d'autre domicile que celui de son mari, lequel en contrepartie est obligé de la recevoir. Elle ne peut effectuer d'actes juridiques sans son autorisation, même si elle est non commune en biens ou séparée de biens (81). La fidélité est réintroduite parmi les devoirs des deux époux, mais là encore la différence de statut joue, puisque l'infidélité est plus sévèrement punie pour les femmes (82) dans la mesure où elle «suppose plus de corruption et des

(76) Ibid, p. 197.

(77) Le Code civil se démarque, en la matière, non seulement du droit privé, mais du droit canonique puisque dans le cadre de ce dernier, le prêtre se borne à assister au mariage en qualité de témoin, mais ne le fait pas. Cf. Julien BoNNECASE (qui reprend l'analyse de M. Planiol), ibid., pp. 153-154.

(78) Le divorce par consentement mutuel est refusé aux époux mineurs; interdit avant 2 ans et après 20 ans de mariage, ainsi que pour les femmes âgées de 45 ans. Cf. Jean-Michel Poughon, Le Code civil, Paris, PUF, Que sais-je ?, p. 34.

(79) Ibid.

(80) Julien BoNNECASE, La philosophie du Code Napoléon..., op. cit., p. 160.

(81) Adrien SIRAMY, Étude sur les origines et les caractères de l'autorisation maritale dans l'histoire du droit français, thèse de droit, Paris, Rousseau, 1901 et Jean Gay, « La capacité de la femme mariée et la puissance maritale dans l'élaboration du Code civil ", Revue de l'Institut Napoléon, $\mathrm{n}^{\circ}$ 161, 1993.

(82) Le code pénal de 1810 prévoit une peine de 3 mois à 2 ans d'emprisonnement pour la femme convaincue d'adultère ainsi que pour son complice (lequel devra payer, en outre, une amende de 100 à 2000 francs); le mari adultère, et à condition qu'il ait entretenu sa concubine sous le toit conjugal, ne sera 
effets plus dangereux » (83) que celle du mari. À la diversité des niveaux statutaires de l'ancien droit se substitue, dans le Code civil, une femme mariée, c'est-à-dire un «statut unique d'ordre public » (84).

Ce nouveau «statut unique» de femme mariée a eu une influence directe sur la manière dont les législateurs napoléoniens ont fait face aux tensions entre famille et nation. Un grand nombre des débats sur le Code ont mis l'accent sur la tension entre la famille et la citoyenneté, notamment à travers le cas de la perte totale des droits de citoyen en cas de mort civile (dont étaient frappés, outre certains criminels, la plupart des émigrés). Les législateurs vont essentiellement discuter de la question de la perte des droits par un chef de famille, se divisant sur les conséquences que cette perte pouvait avoir sur son mariage, notamment sur son autorité sur le reste des membres de la famille. Deux risques étaient envisagés : soit le mariage était automatiquement dissout et dans ce cas, la puissance paternelle était considérée comme un droit civil qui ne pouvait être exercé par quelqu'un privé des droits de citoyen. Le problème, c'est que l'épouse d'un tel homme risquait d'être considérée comme une concubine et ses futurs enfants, comme des bâtards. Soit les liens du mariage étaient maintenus, ce qui signifiait que son épouse et ses enfants risquaient d'être contraints de suivre et d'obéir à un homme par ailleurs exclu de la société.

Les architectes du Code civil avaient une solution évidente à ce dilemme : ils pouvaient laisser au couple le choix de divorcer ou non. Cette solution n'existait pas avant la Révolution : durant l'Ancien Régime, la peine de la mort civile ne mettait pas fin au mariage, dans la mesure où celui-ci était légalement un lien aussi civil que religieux et ne pouvait, par conséquent, être rompu que par la mort. La redéfinition du mariage comme contrat civil, sous la Révolution, accompagnée du droit de divorcer, rendit possible de se séparer d'un conjoint atteint de mort civile : de fait, on a vu qu'afin de prouver leur loyauté à la patrie révolutionnaire, les femmes avaient été requises de divorcer de maris émigrés condamnés à la mort civile.

Les législateurs du Code civil tinrent compte du fait qu'une femme pouvait choisir, du fait de la liberté postulée par la forme contractuelle du mariage, entre sa famille et sa nation, en choisissant de se marier avec un Français ou un étranger. Mais ils limitèrent sérieusement cette «liberté » pour ce qui concernait les conséquences du mariage : ne souhaitant pas exiger des femmes qu'elles agissent de manière «dénaturée » en rompant,

puni que d'une amende de 100 à 2000 francs; il n'y a pas de peine d'emprisonnement prévue pour lui. Et encore, l'adultère du mari faillit ne pas être considéré comme une cause de divorce. Cf. Jean-Michel Poughon, Le Code civil, op. cit., p. 32.

(83) Présentation au corps législatif et exposé des motifs par le conseiller d'État Portalis, séance du 16 ventose an XI (7 mars 1803), dans P. A. FENET, op. cit., t. IX, p. 178.

(84) Michèle BORDEAUX, "Le maître et l'infidèle... », dans La famille, la loi, l'État..., op. cit., p. 435. 
par la demande de divorce, avec leur subordination matrimoniale, ils prirent la décision de faire équivaloir mort civile et dissolution automatique du mariage. L'homme condamné à une mort civile ne pouvait plus être «ni citoyen, ni père, ni époux "; les personnes qui jusqu'alors dépendaient de lui ne pouvaient être requises de choisir entre les liens familiaux et les droits de citoyen français (85).

On retrouve, dans les lois sur l'adoption, le même découplage napoléonien entre famille et nation. Napoléon avait d'abord imaginé l'adoption comme une «espèce de nouveau sacrement » et souhaité que le pouvoir de ratifier celle-ci réside uniquement dans la loi. Il y a là une certaine continuité avec les notions que nous avons déjà abordées d'un roi-père créant de nouveaux citoyens et de nouveaux enfants, puis de l'Assemblée nationale comme "père de la nation ». Mais cette idée fut abandonnée en faveur d'une adoption comme simple contrat privé entre adultes (ce qui signifie qu'un mineur ne pouvait plus être adopté), un contrat d'ailleurs limité puisque seuls les couples sans enfants et âgés de plus de 50 ans pouvaient adopter (86). Cette politique marque l'abandon définitif de l'idée d'adoption nationale autrement que comme titre purement honorifique.

En outre, on se souvient que dans la Constitution de 1793, l'adoption était pensée comme un moyen pour un étranger de devenir citoyen français (parmi d'autres moyens tels que le soin à un vieillard ou le mariage avec une Française). Désormais, le Code civil exclut les étrangers du droit d'adoption et de tutelle (il faudra attendre 1923 pour que ce droit leur soit de nouveau accordé). En outre, l'adoption d'enfants étrangers est limitée : seuls les enfants natifs des pays tolérant l'adoption ou déjà français sont susceptibles d'être adoptés en France. Le lien familial n'est définitivement plus un moyen d'intégration des étrangers à la «grande famille » française; il s'agit, dans l'esprit napoléonien, de protéger au contraire les petites familles des intrus et protéger les enfants de la perte potentielle de leurs droits par la mise sous autorité ou tutelle d'un paterfamilias étranger.

\section{Conclusion}

D'implicite et floue qu'elle était aux débuts de la Révolution, fondée sur un vieil amalgame entre famille et société politique, la mise à l'écart des femmes trouve, dans ce monument qu'est le Code, mais aussi dans ces lois qui touchent à la participation politique et à l'appartenance nationale, une

(85) Alexandre Gaspard GaRY, « Discussion devant le Corps législatif (17 ventose an XI) » dans FENET, Recueil Complet, vol. 7, p. 660.

(86) Éric Andrew GoODHEART, Adoption in the Discourses of the French Revolution, Ph. D. diss., Harvard University, 1997. 
véritable cohérence doctrinale. Cette cohérence est en partie le fruit des efforts pour remédier aux flottements induits par les confusions initiales entre famille et nation. Si le contrat de mariage est bien devenu le fruit de deux volontés libres et égales, l'état de famille, lui, est resté une destinée plus qu'un choix. La distinction qui s'est peu à peu opérée entre ces deux formes de construction politique, l'une volontariste et juridique, l'autre fatale et naturelle, est ce qui a permis de ne plus laisser famille et nation entrer en contradiction. Cette cohérence n'est pas seulement le résultat d'un long travail de recomposition des catégories juridiques : car elle est déjà là, de manière implicite et dispersée, dans quelques textes de loi des premières années de la Révolution. C'est pourquoi la cohérence obtenue à la fin des années 1790 peut être vue comme une forme d'explicitation des normes qui, depuis les États généraux, ont guidé les révolutionnaires dans leur œuvre législative : ces normes, nous les avons appelées «familialistes», afin de bien mettre en évidence le caractère tout à fait spécifique de la définition moderne de l'individu citoyen, qui n'a que peu à voir avec celle que nous lui donnons aujourd'hui (87). Sur cette base, on peut continuer de parler d'une conception individualiste de la citoyenneté, mais à condition d'admettre que cet individu est uniquement celui qui trouve dans une hiérarchie familiale "naturelle " à la fois le fondement et la justification de son autorité spécifique de citoyen (88). Cette hiérarchie naturelle est à l'œuvre dès 1789 , mais à l'insu, la plupart du temps, des législateurs, plus soucieux d'édicter les lois que d'en définir les catégories; attachés à refonder et cherchant avant tout à libérer l'individu, ceux-ci ont d'abord opéré sur les raisons, les supports de la tyrannie, au premier rang desquels l'autorité dans la famille, sans voir toutefois qu'ainsi ils travaillaient des catégories révolutionnaires d'une manière tout à fait traditionnelle, d'une part, et qu'en laissant les femmes hors de la participation électorale légale, ils se fermaient d'emblée un certain nombre de portes. C'est bien dans ce double aveuglement que se situe l'explication du renversement des politiques concernant la famille et la citoyenneté : dans la volonté politique de régénération fondée sur l'individualisation (la démocratisation) de la famille et le maintien non questionné d'évidences quant à l'exercice de l'autorité politique; dans la volonté d'en finir avec l'autorité

(87) De ce paterfamilias qui fonde la Déclaration des droits de l'homme et du citoyen à l'individu sexuellement neutre sur lequel repose notre conception contemporaine de la citoyenneté, il a fallu l'évolution de presque deux cents ans des catégories de la pensée politique.

(88) C'est pourquoi nous ne souscrivons pas pleinement aux analyses qui font de la famille, pendant la période révolutionnaire, soit une simple métaphore du lien politique (Michel BORGETTo, « Métaphore de la famille...", op. cit.) soit un roman, changeant qui plus est, de l'inconscient collectif (cf. Lynn HuNT, Le roman familial..., op. cit). Loin de n'être qu'une image, la famille est une catégorie implicite de la pensée politique qui, comme telle, a des conséquences sur les situations juridiques des personnes; en outre, en tant que catégorie implicite, elle est peu à même d'évoluer, même dans la temporalité exceptionnelle que constitue la période révolutionnaire. C'est ainsi que comme catégorie de pensée elle reste intouchée tout au long du processus révolutionnaire. 
d'un seul, qu'elle soit politique ou domestique, tout en continuant à penser l'exercice individuel de la citoyenneté sur la base d'une capacité politique signalée par le seul état de famille des personnes.

Pour mettre un terme à cet aveuglement initial, il aura fallu l'émergence de ces multiples conflits dont nous avons tenté ici de faire une synthèse. Conflits multiples parce que nombreux, mais aussi parce que divers dans leur nature : l'ambiguité terminologique n'est jamais absente des premières lois sur la citoyenneté, ne serait-ce que parce qu'elles oublient systématiquement de préciser que nulle femme ne saurait être admise à l'exercice du droit de vote - d'où ces conflits entre la norme implicite et les diverses pratiques locales; l'intrusion dans les « liens les plus tendres » d'une violence du soupçon qui ne veut ni ne peut plus opérer de distinction entre la cité et une sphère qui serait protégée contribue sans doute à faire ressentir le besoin d'un nouvel ordre social reconnaissant le principe de séparation entre le public (libre) et le domestique (protégé); enfin, ultime conflit, celui entre les droits de la grande famille et les devoirs de la petite, qui donnera son plein effet à travers les nombreux cas d'épouses et de mères tenues de ne pouvoir défendre leurs droits qu'au nom de leurs devoirs et qui trouvera sa résolution dans la distinction entre les individus de la société civile et politique et les membres de la famille restant sous l'autorité des premiers; situant ainsi d'une manière définitive et non ambiguë les uns et les autres.

Jennifer HEUER

Department of History Middlebury College Middlebury, VT 05753

USA

heuer@pobox.com

Anne VerJus

GAPP-ENS de Cachan

61, Avenue du Président Wilson

94235 Cachan Cedex verjus.anne@wanadoo.fr 